OPEN ACCESS

Edited by:

Junjie Yang,

University of Alabama at Birmingham, United States

Reviewed by:

Chaoshan Han

University of Alabama at Birmingham, United States

$\mathrm{NaXu}$,

Heart Institute, Cincinnati Children's Hospital Medical Center, United States

*Correspondence: Jun-Jie Zhang jameszl|@163.com Shao-Liang Chen chmengx@126.com

Specialty section: This article was submitted to Cardiovascular Biologics and Regenerative Medicine, a section of the journal Frontiers in Cardiovascular Medicine

Received: 06 April 2021 Accepted: 11 May 2021

Published: 31 May 2021

Citation:

Chen A-Q, Gao X-F, Wang Z-M, Wang F, Luo S, Gu Y, Zhang J-J and Chen S-L (2021) Therapeutic

Exosomes in Prognosis and Developments of Coronary Artery

Disease.

Front. Cardiovasc. Med. 8:691548 doi: 10.3389/fcvm.2021.691548

\section{Therapeutic Exosomes in Prognosis and Developments of Coronary Artery Disease}

\author{
Ai-Qun Chen ${ }^{1}$, Xiao-Fei Gao ${ }^{1,2}$, Zhi-Mei Wang ${ }^{1}$, Feng Wang ${ }^{1}$, Shuai Luo ${ }^{1}$, Yue Gu ${ }^{1}$, \\ Jun-Jie Zhang ${ }^{1,2 *}$ and Shao-Liang Chen ${ }^{1,2 *}$ \\ 'Department of Cardiology, Nanjing First Hospital, Nanjing Medical University, Nanjing, China, ${ }^{2}$ Department of Cardiology, \\ Nanjing Heart Centre, Nanjing, China
}

Exosomes, with an diameter of $30 \sim 150 \mathrm{~nm}$, could be released from almost all types of cells, which contain diverse effective constituent, such as RNAs, proteins, lipids, and so on. In recent years, exosomes have been verified to play an important role in mechanism, diagnosis, treatment, and prognosis of cardiovascular disease, especially coronary artery disease (CAD). Moreover, it has also been shown that exosomes derived from different cell types have various biological functions based on the cell stimulation and microenvironment. However, therapeutic exosomes are currently far away from clinical translation, despite it is full of hope. In this review, we summarize an update of the recent studies and systematic knowledge of therapeutic exosomes in atherosclerosis, myocardial infarction, and in-stent restenosis, which might provide a novel insight into the treatment of CAD and promote the potential clinical application of therapeutic exosomes.

Keywords: exosomes, CAD, atherosclerosis, myocardial infarction, drug delivery

\section{INTRODUCTION}

Coronary artery disease (CAD) still remains a high-prevalence, high-risk, and high-fatality cardiovascular disease worldwide. In spite of the profound development of device and agents in $\mathrm{CAD}$ treatment, the prognosis of CAD, especially acute myocardial infarction, is far from being satisfactory $(1,2)$. Recently, exosome emerges as a novel, full of hope, and potential alternative to cell-based therapies of CAD due to its cardioprotective properties (3).

Exosomes, with diameter of $30 \sim 150 \mathrm{~nm}$ and density of $1.13 \sim 1.19 \mathrm{~g} / \mathrm{ml}$, are the smallest extracellular vesicles (EVs) (4), with a bilayer membrane structure released by almost all types of cells $(5,6)$. The biogenesis of exosomes triggers from membrane proteins being endocytosed via inward budding of the cell membrane, which are then transferred to early endosomes (EEs). Afterwards, the EEs mature into multivesicle bodies (MVBs), filled with numerous intraluminal vesicles (ILVs) $(7,8)$, which incorporate proteins, lipids, and genetic material during invagination (9). Finally, MVBs can fuse with cell membrane and release ILVs to the extracellular space (10), as we call them exosomes, or result in degradation via fusing with lysosomes (Figure 1) (11).

However, therapeutic exosomes are currently far away from clinical application, in spite of so many outstanding qualities of exosomes. In this review, we will summarize an update of the recent findings and systematic knowledge of therapeutic exosomes in CAD, which might provide a novel insight into the treatment of CAD and promote the potential clinical translation of therapeutic exosomes. 


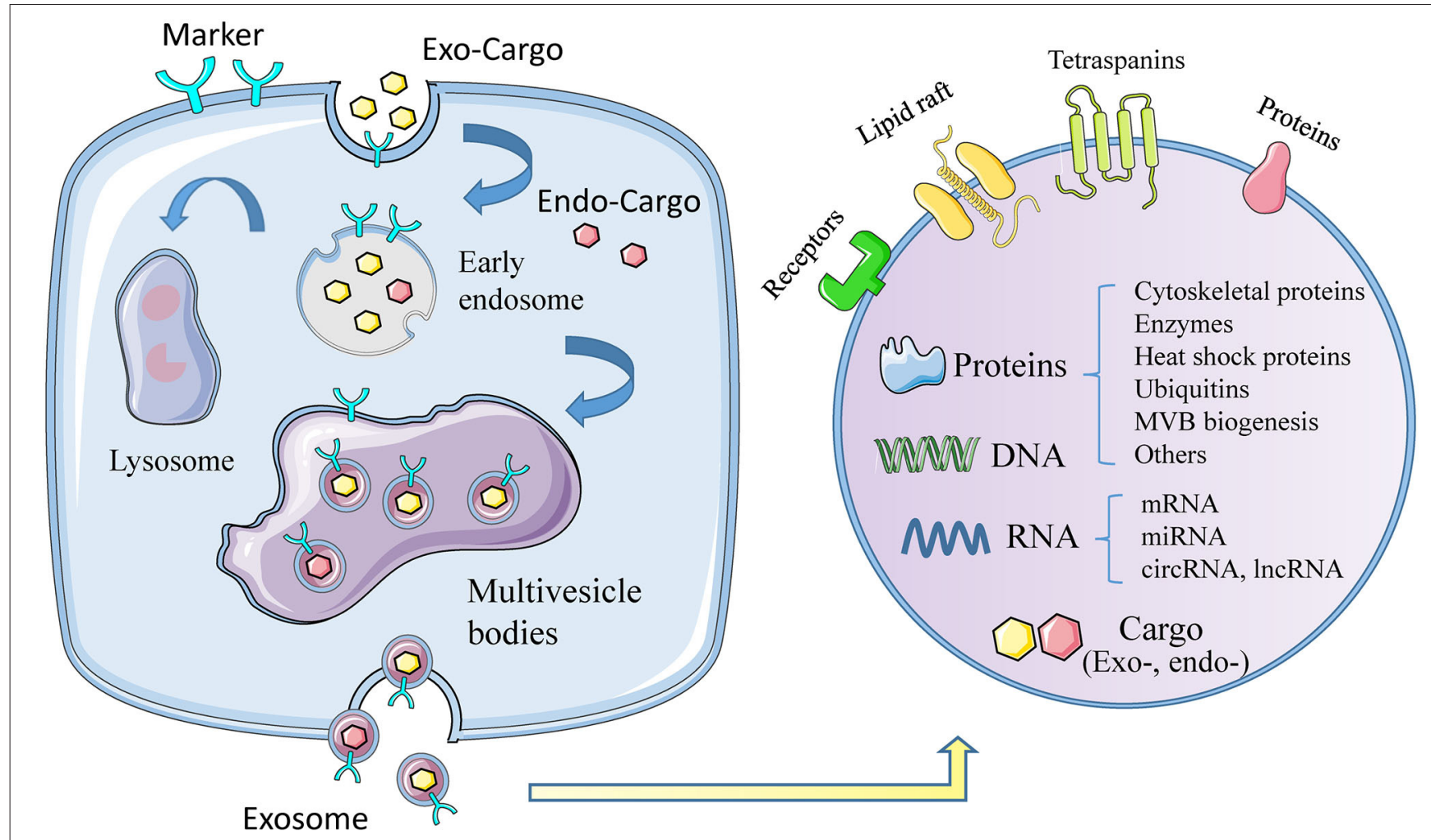

FIGURE 1 | The biogenesis, formation, and content of exosomes. The formation of exosomes begins with invagination of the plasma membrane, and then forms early exosomes, which form multiple vesicles after fusion, and finally release the exosomes out of the cell. During the formation of exosomes, it will actively or passively carry exogenous or endogenous cargoes. The picture on the right shows the membrane structure of exosomes, the contents of exosomes, including proteins, DNA, RNA, and others.

\section{EXOSOMES AND CAD}

According to the progress of CAD, the relationships between exosomes and CAD are summarized into three parts: exosomes in the prevention of atherosclerosis, exosomes in the diagnosis and treatment of myocardial infarction, and exosomes in the development of in-stent restenosis (Table 1).

\section{Therapeutic Exosomes in Atherosclerosis}

A basic progress in the development of atherosclerosis is monocytes/macrophages accumulation into the vessel wall to produce pro-inflammatory cytokines (32). It has been reported that molecularly engineered M2 macrophage-derived exosomes (Further electroporated with hexyl 5-aminolevulinate hydrochloride) alleviated inflammation by promoting the release of anti-inflammatory cytokines (33). Paeonol could restrict atherosclerosis by obviously increasing miR-223 expression in exosomes from monocytes and inhibiting STAT3 pathway (34). Exosomes laden with heat shock protein 27 (HSP27) significantly stimulated NF- $\mathrm{B}$ activation and IL-10 release, suggesting that exosomes could act as a vector in anti-inflammatory therapy (35). Mitochondria constituted a major subset of extracellular vesicles released by LPS-activated monocytes in vitro, which were associated with type I IFN and TNF signaling (36). Exosomes from nicotine-stimulated macrophages could promote atherosclerosis through facilitating VSMC migration and proliferation by targeting miR-21-3p/PTEN (37). Moreover, helicobacter pylori-infected gastric epithelial cellsderived exosomes accelerated macrophage foam cell formation and promoted atherosclerosis by CagA (38). Insulin resistance adipocyte-derived exosomes (IRADEs) has been reported to aggravate the plaque burden, whereas its effect could be attenuated by silencing sonic hedgehog in IRADEs (12). Besides, Jiang et al. (13) also reported that steatotic hepatocyte-derived EVs promoted endothelial inflammation by miR-1 delivery, KLF4 suppression and the NF- $\kappa \mathrm{B}$ pathway activation. And in this instance, exosome therapy might be the reduction of negative contents in exosomes such as miR-1 instead of increasing therapeutic exosomes.

\section{Therapeutic Exosomes in Myocardial Infarction}

Myocardial infarction, which often results in poor clinical outcomes, still remains the lack of effective treatment, especially for those without culprit vessel revascularization (14). Therefore, current clinical treatments are mostly based on easinesss of symptoms rather than repairing infarcted cardiomyocyte (15). 
TABLE 1 | Relationship between exosomes and CAD.

\begin{tabular}{|c|c|c|c|c|c|c|}
\hline Disease & Exosomal cargo & Parent cells & Recipient cells & Target & Biological/clinical relevance & Reference \\
\hline \multirow[t]{7}{*}{ AS } & miR-223 & THP-1 monocyte & HUVEC & STAT-3 pathway & Anti-inflammation & $(12)$ \\
\hline & HSP27 & THP-1 monocyte & - & $N F-\kappa B, I L-10$ & Anti-inflammation & (13) \\
\hline & Mitochondria & Monocyte & Endothelial cell & IFN, TNF & Anti-inflammation & $(14)$ \\
\hline & $\mathrm{miR}-1$ & Hepatocyte & Endothelial cell & $\mathrm{KLF} 4, \mathrm{NF}-\kappa \mathrm{B}$ & Anti-inflammation & $(15)$ \\
\hline & miR-21-3p & MACROPHAGE & VSMC & PTEN & Promote VSMC proliferation and degradation & $(16)$ \\
\hline & - & Gastric epithelial cell & Macrophage & CagA & Promote foam cell formation & $(17)$ \\
\hline & Sonic hedgehog & Adipocyte & HUVECs, MAECs & TGF- $\alpha, I L-1 \beta, I L-6$ & Reduce plaque vulnerability & $(18)$ \\
\hline \multirow[t]{11}{*}{$\mathrm{Ml}$} & miR-342-5p & Endothelials & CMs & Caspase9, Jnk2, Akt & Anti-apoptsis/proliferation & (19) \\
\hline & miR-21 & HEK293T cell & CMS, HUVECS & PDCD4 & Anti-apoptosis & (20) \\
\hline & $\operatorname{miR}-125 b-5 p$ & MSC & CMs & p53, BAK1 & Anti-apoptosis & $(21)$ \\
\hline & miR-210 & EPC & Endothelial cell & Mitochondria & Anti-apoptosis/promote angiogenic function & $(22)$ \\
\hline & miR-24 & Serum & H9c2 cell & Bim & Mediate Remote ischemic preconditioning & (23) \\
\hline & miR-93-5p & Adipose stromal cell & CMs & Atg7, TLR4 & Inhibit autophagy, anti-inflammatory & $(24)$ \\
\hline & IncR & - & Fibroblast, CMs & Neat1 & Anti-fibrosis & (25) \\
\hline & miR-24 & MSC & $\mathrm{CD} 8+\mathrm{T}$ & Bim & Anti-fibrosis & $(26)$ \\
\hline & miR-130-3p & Adipocyte & CMs & AMPK $\alpha 1 / \alpha 2$, Birc6, and Ucp3 & Anti-apoptosis (diabetic) & \\
\hline & Cytotoxic substance & Serum & $\mathrm{HL}-1 \mathrm{CMs}$ & Compliment C4, ApoE, Apo C-IV & Anti-apoptosis (diabetic) & $(27)$ \\
\hline & ILK & Progenitor & $\mathrm{CMs}$ & $N F-\kappa B$ & Enhance myocardial repair & (28) \\
\hline \multirow[t]{3}{*}{ ISR } & miR-222 & M1-macrophages & VSMC & CDKN1B/CDKN1C & Promote VSMC proliferation and degradation & $(29)$ \\
\hline & miR-125b & MSC & VSMC & Myosin-1E & Promote VSMC proliferation and degradation & $(30)$ \\
\hline & miR-21-5p & EPC & HUVEC & THBS1 & Promote repair of endothelial cells & (31) \\
\hline
\end{tabular}

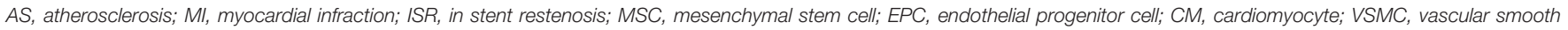
muscle cell; HUVEC, human umbilical vein endothelial cell.

Exosomes reveal significant anti-apoptosis of cardiomyocyte after myocardial infarction. Exercise-derived exosomal miR-342$5 \mathrm{p}$ inhibited cardiomyocyte apoptosis by targeting Caspase 9 and Jnk2 after left anterior descending artery occlusion (16). EVs overexpressing miR-21 could dramatically reduce PDCD4 expression and alleviate myocardial apoptosis (15). Hypoxia-conditioned bone marrow-mesenchymal stem cells (MSCs)-derived exosomes (Hypo-Exo) could also protect cardiomyocytes from apoptosis by enrichment of miR-125b$5 \mathrm{p}$ and suppressing the expression of genes $p 53$ and BAK1 (17). In addition, miR-210 in endothelial progenitor cell-derived exosomes (EPC-EXs) possessed antiapoptotic functions onto hypoxia/reoxygenation-injured human endothelial cells (18). Remote ischemic preconditioning-induced exosomes (RIPCExo) also could transfer miR-24 into myocardium to inhibit apoptosis (39).

Exosomes also provide cardioprotection by activating cell survival signals, inhibiting inflammatory factors, delaying ventricular remodeling, and reducing myocardial fibrosis after the occurrence of myocardial infarction. Exercise-derived exosome (Ex-exo) could carry miR-342-5p to promote Akt phosphorylation by targeting gene Ppmlf (16). MiR-93-5p in adipose stromal cell-derived exosomes (ADSC-Exo) inhibited inflammatory response and prevented myocardial infarction by targeting Atg7 and TLR4 (20). Kenneweg et al. (19) had reported that fibroblasts absorbed lncR-EVs and promoted myocardial fibrosis by targeting Neat1. Moreover, exosomal miR-24, derived from allogenic human umbilical MSC, could inhibit cardiac fibrosis (21).

Patients suffering from myocardial infarction often have a history of diabetes. Gan et al. (22) had demonstrated that the enrichment of miR-130b-3p from dysfunctional adipocyte exacerbated myocardial infarction and cardiomyocyte apoptosis. Serum-exosomes from normoglycemic rats could alleviate the death of hypoxia/reoxygenation-induced $H L-1$ cell, however, which disappears in type-2 diabetes rat model (23).

Exosomes also can serve as an adjuvant therapy. Integrin Linked Kinase (ILK) acted as a target kinase by which progenitor cell-derived exosomes attenuated myocardial injury (24). Cheng et al. (25) have reported that miRNA in EVs contributed to early detection of CAD by means of point-of care applications.

\section{Therapeutic Exosomes in In-stent Restenosis}

Percutaneous coronary intervention has become a very important treatment strategy for CAD, but in-stent restenosis is blamed for the main cause of stent failure in patients with CAD $(26,40)$. Several previous studies have shown that the risk of in-stent restenosis in CAD patients undergoing coronary stent implantation during 1 year follow-up was $\sim 5-10 \%$ (27). The underlying mechanisms of in-stent restenosis are quite complex, and at least exosomes play a crucial role in the development of in-stent restenosis. For example, miR-222 from M1 macrophages (M1M)-derived exosomes promoted vascular smooth muscle 


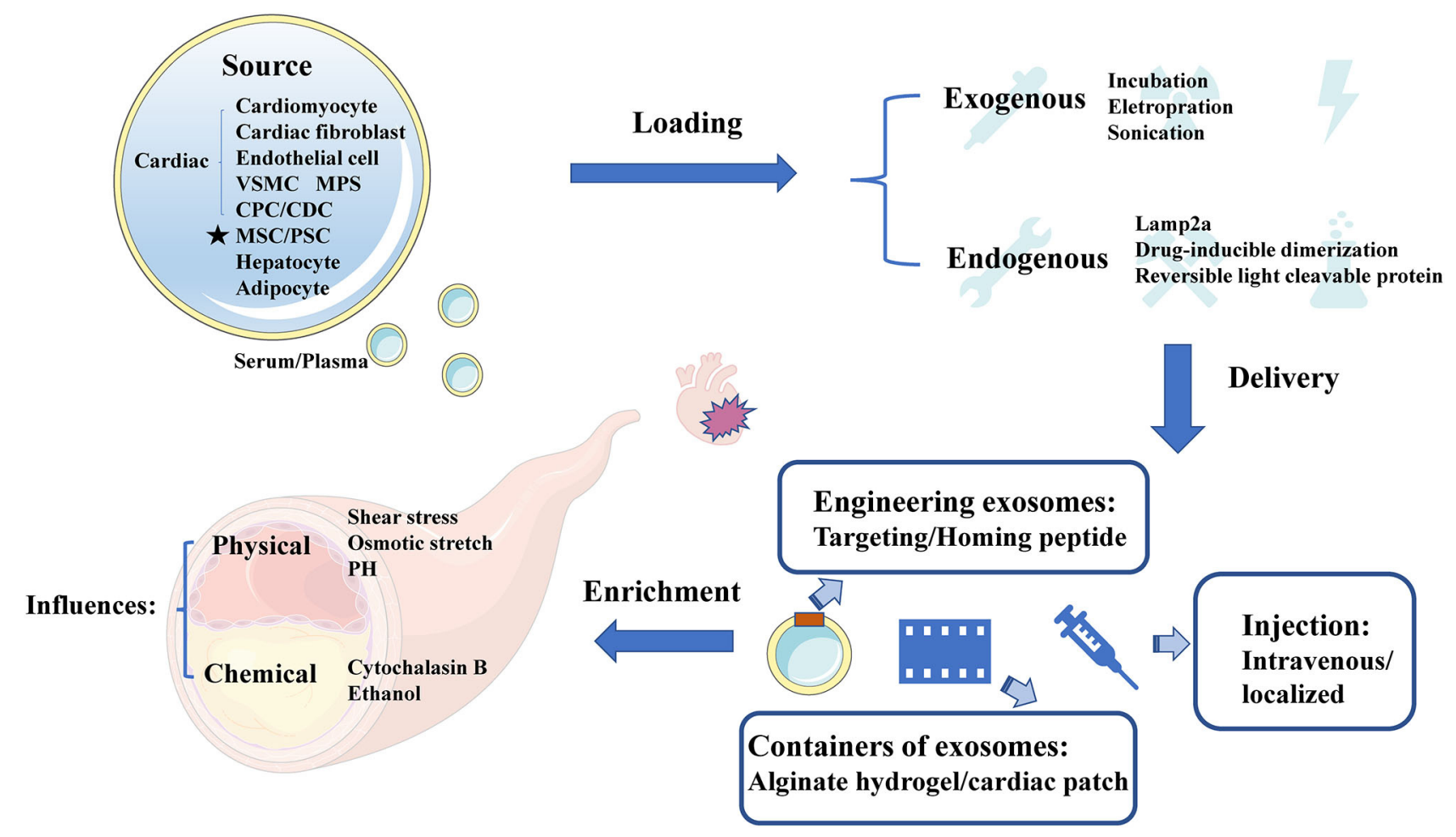

FIGURE 2 | The sources, cargo loading, delivery, and enrichment of therapeutic exosomes. Therapeutic exosomes originate from a variety of cells, including some derived from cardiovascular cells, stem cells, and others. Next, we introduce the method of carrying endogenous or exogenous goods. It then summarizes the optimization strategies for exosome delivery, including targeting peptides, novel exosome containers, and injection methods for exosomes. Finally, we analyze the influencing factors of the enrichment efficiency of exosomes. VSMC, vascular smooth muscle cell; MPS, mononuclear phagocyte system; CPC, cardiac progenitor cell; CDC, cardiosphere-derived cells; MSC, mesenchymal stem cell; PSC, pluripotent stem cell; Cltc, clathrin heavy chain.

cells (VSMCs) proliferation and migration, which resulted in restenosis (41). Wang et al. (42) reported that MSC-Exo enriched miR-125b and inhibited the proliferation and migration of VSMC by targeting myosin 1E. Moreover, EPC-Exo also were involved in the prevention of restenosis through delivering miR-21-5p and inhibiting THBS1 expression (43). Recently, exosome-eluting stents have been proven to reduce intimal hyperplasia and accelerate re-endothelialization in the ischemic injury rat model.

\section{OPTIMIZED TREATMENT STRATEGY}

Exosomes appear superiority and irreplaceable biological functions, and the clinical application of therapeutic exosomes is full of hope. In the first place, exosomes can avoid phagocytosis and bypass the engulfment by lysosomes (44) to exhibit a longer circulation half-life due to the protection of phospholipid bilayer membrane (28). Secondly, phospholipid bilayer of exosomes is also beneficial to the fusion with membrane of recipient cells (29). Thirdly, exosomes derived from animals or patients have the high homolog and low immune response to avoid exosomes degradation (30). Finally, exosomal regulation of "Homing" effect has been reported to target the cell type where exosomes were produced (31), which can provide a shortcut for exosomes delivery. In need of optimized treatment strategy, we summarized the latest research involved of sources, cargo loading, delivery and enrichment of therapeutic exosomes (Figure 2).

\section{Source of Therapeutic Exosomes}

It has been reported that the sources of CAD related therapeutic exosomes were commonly cardiovascular-derived endothelial cells, smooth muscle cells, macrophages and cardiac fibroblasts (45). In recent years, several studies have highlighted the value of MSC-Exo therapy in cardiac protection $(46,47)$, and MSC could secret the highest amount of exosomes (48). Moreover, other studies found that circulating-Exo, adipocyte-EVs (12), hepatocyte-EVs (13), accompanied with different degrees of heterogeneity, all existed therapeutically effect upon CAD.

\section{Loading Therapeutic Cargo in Exosomes}

Although many therapeutic cargoes are inherent in parent cells previously, some therapeutic cargoes could only be loaded into exosomes by artificial means. Normally, cargoes could be loaded through fusion with liposomes, adsorption of molecules to the surface of exosomes and the insertion of lipids (49). It has been reported that a few procedures, such as incubation, electroporation (33), sonication (50), and so on (51), could promote cargo loading. When choosing the loading method of 
cargoes, we should consider the loading efficiency (52), and whether this loading method will change the physical and chemical characteristics of exosomes (53). Besides, membrane protein Lamp2a could increase the loading of miRNA into EVs (54). Moreover, drug-inducible dimerization (55), reversible light cleavable protein (56), and several advanced means of engineering exosomes also contribute to the loading of endogenous cargoes.

\section{Delivery Method}

Normally, therapeutic exosomes were injected intravenously and act on the cardiovascular diseases through the circulatory system as an essential treatment. However, most of these exosomes are taken up by liver or spleen (57). Loading homing peptides has become a popular way to optimize delivery of exosomes (58). In cardiovascular field, several homing peptides in connection with atherosclerosis $(59,60)$, and ischemia/reperfusion-injured cardiomyocytes (61) have been identified and applied in therapeutic regimen. For example, Wang et al. (62) have demonstrated that engineered exosomes fused with ischemic myocardium-targeting peptide (IMTP) increasingly accumulated in ischemic heart area. Furthermore, it has been reported that exosomes conjugated with cardiac homing peptide (CHP) has higher retention in infarcted heart (63).

Besides, Song et al. (15) have reported that localized injection of EVs attenuated the apoptosis of cardiomyocytes and endothelial cells in a preclinical myocardial infarction (MI) animal model. To reduce losses during transportation, Lv et al. (64) have reported that sEVs, incorporated in alginate hydrogel, act as a new regimen of therapy. An off-the-shelf therapeutic cardiac patch, composed of extracellular matrix and cardiac stromal cells (CSC), has been confirmed in the model of MI (65). The examples above demonstrate the superiority of local delivery of exosomes and improve the retention rate of exosomes.

\section{Enrichment Efficiency}

The enrichment efficiency of exosomes is affected by physical and chemical stimuli. The physical stimulation of exosomes mainly includes shear stress, osmotic stretch, $\mathrm{PH}$ and others (66). More importantly, the change of blood flow shear force, as the initiating factor of coronary artery disease, has also become a difficult problem for exosome delivery. Here, we focus on the shear stress in vessel where exosomes were regulated. While shear stress remain within 1-70 dynes $/ \mathrm{cm}^{2}$ in normal blood vessels, severely narrowed blood vessels can produce over 1,000 dynes $/ \mathrm{cm}^{2}$ (67). High shear stress, occurring in atherosclerotic arteries, could accelerate the release

\section{REFERENCES}

1. Sousa-Uva M, Neumann FJ, Ahlsson A, Alfonso F, Banning AP, Benedetto U, et al. 2018 ESC/EACTS Guidelines on myocardial revascularization. Eur J Cardio Thorac Surg. (2019) 55:4-90. doi: 10.1093/ejcts/ ezy289

2. Benjamin EJ, Muntner P, Alonso A, Bittencourt MS, Callaway CW, Carson AP, et al. Heart disease and stroke statistics-2019 update: a of circulating-EVs gradually (68). The mechanisms of shear stress on EVs secretion relate to the response of membrane tension (69). Besides, calcium could enhance exosomes secretion from a microenvironment perspective (70), whereas arterial hypertension was also associated with the increase of shear stress from a macro perspective (71). Evidence proved that exercise training could increase EVs release under high shear stress, and decrease the risk of thrombosis correspond to stenotic arteries (72). Exosomes could also be affected by chemical trigger, including cytochalasin B and ethanol (46).

\section{CONCLUSION AND FUTURE PERSPECTIVE}

In recent years, the therapeutic effect of exosomes on heart diseases has been gradually discovered. We have summarized the progress in studying exosomes as drug delivery vehicles. Before entering the clinical transformation, a perfect therapeutic concept of exosomes is essential (3), and pioneering in the field of exosomes is tumor-related studies. We can draw on tumor-related studies to optimize treatment regimens. Certainly, CAD-targeted treatment options also need to take notice of the cardiovascular lineage specificity.

Exosomes, as natural drug delivery vehicles, have excellent biocompatibility and targeting properties. We have discovered the potential of exosomes in the treatment of CAD based on existing research. However, exosomes still face huge resistance in clinical transformation. Moreover, we hope that the optimization of therapeutic exosomes is getting better and enter the clinical application stage as soon as possible.

\section{AUTHOR CONTRIBUTIONS}

A-QC and X-FG wrote the manuscript. Z-MW and FW prepared the figures. SL and YG prepared the table. J-JZ and S-LC provided the idea and revised the manuscript. All authors have agreed to the published version of the manuscript.

\section{FUNDING}

This study was funded by the National Natural Science Foundation of China (NSFC 81970307), and was jointly supported by Six Talent Peaks Project of Jiangsu Province (2019-WSN-156), Social Development Project of Jiangsu Province (BE2019615, BE2019616), Jiangsu Commission of Health (H2019077), and Nanjing Health Youth Talent Training project (QRX17017).

3. Nazari-Shafti TZ, Stamm C, Falk V, Emmert MY. Exosomes for cardioprotection: are we ready for clinical translation? Eur Heart J. (2019). 40:953-6. doi: 10.1093/eurheartj/ehz106

4. Shao H, Im H, Castro CM, Breakefield X, Weissleder R, Lee H. New technologies for analysis of extracellular vesicles. Chem Rev. (2018) 118:191750. doi: 10.1021/acs.chemrev.7b00534 
5. Théry C, Zitvogel L, Amigorena S. Exosomes: composition, biogenesis and function. Nat Rev Immunol. (2002) 2:569-79. doi: 10.1038/nri855

6. Kalluri R, LeBleu VS. The biology, function, and biomedical applications of exosomes. Science. (2020) 367:eaau6977. doi: 10.1126/science.aau6977

7. Février B, Raposo G. Exosomes: endosomal-derived vesicles shipping extracellular messages. Curr Opin Cell Biol. (2004) 16:415-21. doi: 10.1016/j.ceb.2004.06.003

8. Merchant ML, Rood IM, Deegens JKJ, Klein JB. Isolation and characterization of urinary extracellular vesicles: implications for biomarker discovery. Nat Rev Nephrol. (2017) 13:731-49. doi: 10.1038/nrneph.2017.148

9. van Niel G, D'Angelo G, Raposo G. Shedding light on the cell biology of extracellular vesicles. Nat Rev Mol Cell Biol. (2018) 19:21328. doi: $10.1038 / \mathrm{nrm} .2017 .125$

10. Lo Cicero A, Stahl PD, Raposo G. Extracellular vesicles shuffling intercellular messages: for good or for bad. Curr Opin Cell Biol. (2015). 35:6977. doi: 10.1016/j.ceb.2015.04.013

11. Raiborg C, Stenmark H. The ESCRT machinery in endosomal sorting of ubiquitylated membrane proteins. Nature. (2009) 458:445-52. doi: 10.1038/nature07961

12. Wang F, Chen FF, Shang YY, Li Y, Wang ZH, Han L, et al. Insulin resistance adipocyte-derived exosomes aggravate atherosclerosis by increasing vasa vasorum angiogenesis in diabetic ApoE(-/-) mice. Int J Cardiol. (2018) 265:181-7. doi: 10.1016/j.ijcard.2018.04.028

13. Jiang F, Chen Q, Wang W, Ling Y, Yan Y, Xia P. Hepatocyte-derived extracellular vesicles promote endothelial inflammation and atherogenesis via microRNA-1. J Hepatol. (2020) 72:156-66. doi: 10.1016/j.jhep.2019.09.014

14. Rao SV, Kaul P, Newby LK, Lincoff AM, Hochman J, Harrington RA, et al. Poverty, process of care, and outcome in acute coronary syndromes. J Am Coll Cardiol. (2003) 41:1948-54. doi: 10.1016/S0735-1097(03)00402-9

15. Song Y, Zhang C, Zhang J, Jiao Z, Dong N, Wang G, et al. Localized injection of miRNA-21-enriched extracellular vesicles effectively restores cardiac function after myocardial infarction. Theranostics. (2019) 9:234660. doi: 10.7150/thno.29945

16. Hou Z, Qin X, Hu Y, Zhang X, Li G, Wu J, et al. Longterm exercise-derived exosomal miR-342-5p: a novel exerkine for cardioprotection. Circ Res. (2019) 124:1386-400. doi: 10.1161/CIRCRESAHA.118.314635

17. Zhu LP, Tian T, Wang JY, He JN, Chen T, Pan M, et al. Hypoxia-elicited mesenchymal stem cell-derived exosomes facilitates cardiac repair through miR-125b-mediated prevention of cell death in myocardial infarction. Theranostics. (2018) 8:6163-77. doi: 10.7150/thno.28021

18. Ma X, Wang J, Li J, Ma C, Chen S, Lei W, et al. Loading MiR-210 in endothelial progenitor cells derived exosomes boosts their beneficial effects on hypoxia/reoxygeneation-injured human endothelial cells via protecting mitochondrial function. Cell Physiol Biochem. (2018) 46:66475. doi: 10.1159/000488635

19. Kenneweg F, Bang C, Xiao K, Boulanger CM, Loyer X, Mazlan S, et al. Long noncoding RNA-enriched vesicles secreted by hypoxic cardiomyocytes drive cardiac fibrosis. Mol Ther Nucleic Acids. (2019) 18:363-74. doi: 10.1016/j.omtn.2019.09.003

20. Liu J, Jiang $M$, Deng S, Lu J, Huang $H$, Zhang $Y$, et al. miR-93-5pcontaining exosomes treatment attenuates acute myocardial infarctioninduced myocardial damage. Mol Ther Nucleic Acids. (2018) 11:10315. doi: 10.1016/j.omtn.2018.01.010

21. Shao L, Zhang Y, Pan X, Liu B, Liang C, Zhang Y, et al. Knockout of $\beta-2$ microglobulin enhances cardiac repair by modulating exosome imprinting and inhibiting stem cell-induced immune rejection. Cell Mol Life Sci. (2020) 77:937-52. doi: 10.1007/s00018-019-03220-3

22. Gan L, Xie D, Liu J, Lau WB, Christopher TA, Lopez B, et al. Small extracellular microvesicles mediated pathological communications between dysfunctional adipocytes and cardiomyocytes as a novel mechanisms exacerbating ischemia/reperfusion injury in diabetic mice. Circulation. (2020) 141:968-83. doi: 10.1161/CIRCULATIONAHA.119.042640

23. Wider J, Undyala VVR, Whittaker P, Woods J, Chen X, Przyklenk K. Remote ischemic preconditioning fails to reduce infarct size in the Zucker fatty rat model of type-2 diabetes: role of defective humoral communication. Basic Res Cardiol. (2018) 113:16. doi: 10.1007/s00395-018-0674-1

24. Yue Y, Wang C, Benedict C, Huang G, Truongcao M, Roy R, et al. Interleukin10 deficiency alters endothelial progenitor cell-derived exosome reparative effect on myocardial repair via integrin-linked kinase enrichment. Circ Res. (2020) 126:315-29. doi: 10.1161/CIRCRESAHA.119.315829

25. Cheng HL, Fu CY, Kuo WC, Chen YW, Chen YS, Lee YM, et al. Detecting miRNA biomarkers from extracellular vesicles for cardiovascular disease with a microfluidic system. Lab Chip. (2018) 18:2917-25. doi: 10.1039/C8LC00386F

26. Zhang J, Gao X, Kan J, Ge Z, Han L, Lu S, et al. Intravascular ultrasound versus angiography-guided drug-eluting stent implantation: the ULTIMATE trial. $J$ Am Coll Cardiol. (2018) 72:3126-37. doi: 10.1016/j.jacc.2018.09.013

27. Gao XF, Kan J, Zhang YJ, Zhang JJ, Tian NL, Ye F, et al. Comparison of one-year clinical outcomes between intravascular ultrasound-guided versus angiography-guided implantation of drug-eluting stents for left main lesions: a single-center analysis of a 1,016-patient cohort. Patient Prefer Adherence. (2014) 8:1299-309. doi: 10.2147/PPA.S65768

28. Saunderson SC, Dunn AC, Crocker PR, McLellan AD. CD169 mediates the capture of exosomes in spleen and lymph node. Blood. (2014) 123:20816. doi: 10.1182/blood-2013-03-489732

29. Mathivanan S, Ji H, Simpson RJ. Exosomes: extracellular organelles important in intercellular communication. J Proteomics. (2010) 73:190720. doi: 10.1016/j.jprot.2010.06.006

30. Ha D, Yang N, Nadithe V. Exosomes as therapeutic drug carriers and delivery vehicles across biological membranes: current perspectives and future challenges. Acta Pharm Sin B. (2016) 6:287-96. doi: 10.1016/j.apsb.2016.02.001

31. Park EJ, Prajuabjinda O, Soe ZY, Darkwah S, Appiah MG, Kawamoto E, et al. Exosomal regulation of lymphocyte homing to the gut. Blood Adv. (2019) 3:1-11. doi: 10.1182/bloodadvances.2018024877

32. Libby $\mathrm{P}$, Okamoto $\mathrm{Y}$, Rocha VZ, Folco E. Inflammation in atherosclerosis: transition from theory to practice. Circ J. (2010) 74:213-20. doi: 10.1253/circj.CJ-09-0706

33. Wu G, Zhang J, Zhao Q, Zhuang W, Ding J, Zhang C, et al. Molecularly engineered macrophage-derived exosomes with inflammation tropism and intrinsic heme biosynthesis for atherosclerosis treatment. Angew Chem Int Ed Engl. (2020) 59:4068-74. doi: 10.1002/anie.201913700

34. Liu Y, Li C, Wu H, Xie X, Sun Y, Dai M. Paeonol attenuated inflammatory response of endothelial cells via stimulating monocytesderived exosomal microRNA-223. Front Pharmacol. (2018) 9:1105. doi: 10.3389/fphar.2018.01105

35. Shi C, Ulke-Lemée A, Deng J, Batulan Z, O’Brien ER. Characterization of heat shock protein 27 in extracellular vesicles: a potential antiinflammatory therapy. FASEB J. (2019) 33:1617-30. doi: 10.1096/fj.20180 0987R

36. Puhm F, Afonyushkin T, Resch U, Obermayer G, Rohde M, Penz T, et al Mitochondria are a subset of extracellular vesicles released by activated monocytes and induce type I IFN and TNF responses in endothelial cells. Circ Res. (2019) 125:43-52. doi: 10.1161/CIRCRESAHA.118.3 14601

37. Zhu J, Liu B, Wang Z, Wang D, Ni H, Zhang L, et al. Exosomes from nicotine-stimulated macrophages accelerate atherosclerosis through miR-213p/PTEN-mediated VSMC migration and proliferation. Theranostics. (2019) 9:6901-19. doi: 10.7150/thno.37357

38. Yang S, Xia YP, Luo XY, Chen SL, Li BW, Ye ZM, et al. Exosomal CagA derived from Helicobacter pylori-infected gastric epithelial cells induces macrophage foam cell formation and promotes atherosclerosis. J Mol Cell Cardiol. (2019) 135:40-51. doi: 10.1016/j.yjmcc.2019.07.011

39. Minghua W, Zhijian G, Chahua H, Qiang L, Minxuan X, Luqiao W, et al. Plasma exosomes induced by remote ischaemic preconditioning attenuate myocardial ischaemia/reperfusion injury by transferring miR-24. Cell Death Dis. (2018) 9:320. doi: 10.1038/s41419-018-0274-x

40. Gao XF, Lu S, Ge Z, Zuo GF, Wang ZM, Wang F, et al. Relationship between high platelet reactivity on clopidogrel and long-term clinical outcomes after drug-eluting stents implantation (PAINT-DES): a prospective, propensity score-matched cohort study. BMC Cardiovasc Disord. (2018) 18:103. doi: 10.1186/s12872-018-0841-1

41. Wang Z, Zhu H, Shi H, Zhao H, Gao R, Weng X, et al. Exosomes derived from M1 macrophages aggravate neointimal hyperplasia following carotid artery injuries in mice through miR-222/CDKN1B/CDKN1C pathway. Cell Death Dis. (2019) 10:422. doi: 10.1038/s41419-019-1667-1 
42. Wang D, Gao B, Yue J, Liu F, Liu Y, Fu W, et al. Exosomes from mesenchymal stem cells expressing miR-125b inhibit neointimal hyperplasia via myosin IE. J Cell Mol Med. (2019) 23:1528-40. doi: 10.1111/jcmm.14060

43. Hu H, Wang B, Jiang C, Li R, Zhao J. Endothelial progenitor cell-derived exosomes facilitate vascular endothelial cell repair through shuttling miR21-5p to modulate Thrombospondin-1 expression. Clin Sci (Lond). (2019) 133:1629-44. doi: 10.1042/CS20190188

44. Bunggulawa EJ, Wang W, Yin T, Wang N, Durkan C, Wang Y, et al. Recent advancements in the use of exosomes as drug delivery systems. $J$ Nanobiotechnol. (2018) 16:81. doi: 10.1186/s12951-018-0403-9

45. Chistiakov DA, Orekhov AN, Bobryshev YV. Cardiac extracellular vesicles in normal and infarcted heart. Int J Mol Sci. (2016) 17:63. doi: 10.3390/ijms17010063

46. Piffoux M, Nicolás-Boluda A, Mulens-Arias V, Richard S, Rahmi G, Gazeau F, et al. Extracellular vesicles for personalized medicine: The input of physically triggered production, loading and theranostic properties. Adv Drug Deliv Rev. (2019) 138:247-58. doi: 10.1016/j.addr.2018.12.009

47. Caplan AI, Dennis JE. Mesenchymal stem cells as trophic mediators. J Cell Biochem. (2006) 98:1076-84. doi: 10.1002/jcb.20886

48. Yeo RW, Lai RC, Zhang B, Tan SS, Yin Y, Teh BJ, et al. Mesenchymal stem cell: an efficient mass producer of exosomes for drug delivery. Adv Drug Deliv Rev. (2013) 65:336-41. doi: 10.1016/j.addr.2012.07.001

49. Richter M, Vader P, Fuhrmann G. Approaches to surface engineering of extracellular vesicles. Adv Drug Deliv Rev. (2021) 173:416-26. doi: 10.1016/j.addr.2021.03.020

50. Kim MS, Haney MJ, Zhao Y, Mahajan V, Deygen I, Klyachko NL, et al. Development of exosome-encapsulated paclitaxel to overcome MDR in cancer cells. Nanomed Nanotechnol Biol Med. (2016) 12:65564. doi: 10.1016/j.nano.2015.10.012

51. Haney MJ, Klyachko NL, Zhao Y, Gupta R, Plotnikova EG, He Z, et al. Exosomes as drug delivery vehicles for Parkinson's disease therapy. J Control Release. (2015) 207:18-30. doi: 10.1016/j.jconrel.2015.03.033

52. Elsharkasy OM, Nordin JZ, Hagey DW, de Jong OG, Schiffelers RM, Andaloussi SE, et al. Extracellular vesicles as drug delivery systems: Why and how? Adv Drug Deliv Rev. (2020) 159:332-43. doi: 10.1016/j.addr.2020. 04.004

53. Kooijmans SAA, Stremersch S, Braeckmans K, de Smedt SC, Hendrix A, Wood MJA, et al. Electroporation-induced siRNA precipitation obscures the efficiency of siRNA loading into extracellular vesicles. J Control Release. (2013) 172:229-38. doi: 10.1016/j.jconrel.2013.08.014

54. Sutaria DS, Jiang J, Elgamal OA, Pomeroy SM, Badawi M, Zhu X, et al. Low active loading of cargo into engineered extracellular vesicles results in inefficient miRNA mimic delivery. J Extracell Vesicles. (2017) 6:1333882. doi: 10.1080/20013078.2017.1333882

55. Banaszynski LA, Liu CW, Wandless TJ. Characterization of the FKBP.rapamycin.FRB ternary complex. J Am Chem Soc. (2005) 127:4715-21. doi: 10.1021/ja043277y

56. Yim N, Ryu SW, Choi K, Lee KR, Lee S, Choi H, et al. Exosome engineering for efficient intracellular delivery of soluble proteins using optically reversible protein-protein interaction module. Nat Commun. (2016) 7:12277. doi: $10.1038 /$ ncomms 12277

57. Armstrong JPK, Stevens MM. Strategic design of extracellular vesicle drug delivery systems. Adv Drug Deliv Rev. (2018) 130:12-6. doi: 10.1016/j.addr.2018.06.017

58. Shen $\mathrm{B}, \mathrm{Wu} \mathrm{N}$, Yang JM, Gould SJ. Protein targeting to exosomes/microvesicles by plasma membrane anchors. J Biol Chem. (2011) 286:14383-95. doi: 10.1074/jbc.M110.208660

59. Hong HY, Lee HY, Kwak W, Yoo J, Na MH, So IS, et al. Phage display selection of peptides that home toatherosclerotic plaques: IL-4 receptor as a candidate target in atherosclerosis. J Cell Mol Med. (2008) 12:200314. doi: 10.1111/j.1582-4934.2008.00189.x

60. Lee GY, Kim JH, Oh GT, Lee BH, Kwon IC, Kim IS. Molecular targeting of atherosclerotic plaques by a stabilin-2-specific peptide ligand. J Control Release. (2011) 155:211-7. doi: 10.1016/j.jconrel.2011.07.010

61. Won YW, McGinn AN, Lee M, Bull DA, Kim SW. Targeted gene delivery to ischemic myocardium by homing peptide-guided polymeric carrier. $\mathrm{Mol}$ Pharm. (2013) 10:378-85. doi: 10.1021/mp300500y

62. Wang X, Chen Y, Zhao Z, Meng Q, Yu Y, Sun J, et al. Engineered exosomes with ischemic myocardium-targeting peptide for targeted therapy in myocardial infarction. J Am Heart Assoc. (2018) 7:e008737. doi: 10.1161/JAHA.118.008737

63. Vandergriff A, Huang K, Shen D, Hu S, Hensley MT, Caranasos TG, et al. Targeting regenerative exosomes to myocardial infarction using cardiac homing peptide. Theranostics. (2018) 8:1869-78. doi: 10.7150/thno.20524

64. Lv K, Li Q, Zhang L, Wang Y, Zhong Z, Zhao J, et al. Incorporation of small extracellular vesicles in sodium alginate hydrogel as a novel therapeutic strategy for myocardial infarction. Theranostics. (2019) 9:740316. doi: 10.7150/thno.32637

65. Huang K, Ozpinar EW, Su T, Tang J, Shen D, Qiao L, et al. An off-the-shelf artificial cardiac patch improves cardiac repair after myocardial infarction in rats and pigs. Sci Transl Med. (2020) 12:eaat9683. doi: 10.1126/scitranslmed.aat9683

66. Liu H, Liu S, Qiu X, Yang X, Bao L, Pu F, et al. Donor MSCs release apoptotic bodies to improve myocardial infarction via autophagy regulation in recipient cells. Autophagy. (2020) 16:2140-55. doi: 10.1080/15548627.2020.1717128

67. Korin N, Gounis MJ, Wakhloo AK, Ingber DE. Targeted drug delivery to flow-obstructed blood vessels using mechanically activated nanotherapeutics. JAMA Neurol. (2015) 72:119-22. doi: 10.1001/jamaneurol.2014.2886

68. Miyazaki Y, Nomura S, Miyake T, Kagawa H, Kitada C, Taniguchi $\mathrm{H}$, et al. High shear stress can initiate both platelet aggregation and shedding of procoagulant containing microparticles. Blood. (1996) 88:345664. doi: 10.1182/blood.V88.9.3456.bloodjournal 8893456

69. Staykova M, Holmes DP, Read C, Stone HA. Mechanics of surface area regulation in cells examined with confined lipid membranes. Proc Natl Acad Sci U S A. (2011) 108:9084-8. doi: 10.1073/pnas.1102358108

70. Savina A, Fader CM, Damiani MT, Colombo MI. Rab11 promotes docking and fusion of multivesicular bodies in a calciumdependent manner. Traffic (Copenhagen, Denmark). (2005) 6:131-43. doi: 10.1111/j.1600-0854.2004.00257.x

71. Gatzoulis MA, Alonso-Gonzalez R, Beghetti M. Pulmonary arterial hypertension in paediatric and adult patients with congenital heart disease. Eur Respir Rev. (2009) 18:154-61. doi: 10.1183/09059180.00003309

72. Chen YW, Chen YC, Wang JS. Absolute hypoxic exercise training enhances in vitro thrombin generation by increasing procoagulant platelet-derived microparticles under high shear stress in sedentary men. Clin Sci (Lond). (2013) 124:639-49. doi: 10.1042/CS20120540

Conflict of Interest: The authors declare that the research was conducted in the absence of any commercial or financial relationships that could be construed as a potential conflict of interest.

Copyright (c) 2021 Chen, Gao, Wang, Wang, Luo, Gu, Zhang and Chen. This is an open-access article distributed under the terms of the Creative Commons Attribution License (CC BY). The use, distribution or reproduction in other forums is permitted, provided the original author(s) and the copyright owner(s) are credited and that the original publication in this journal is cited, in accordance with accepted academic practice. No use, distribution or reproduction is permitted which does not comply with these terms. 